\title{
Research on the application of experimental management based on Android mobile phone platform
}

\author{
Chunxi Zhao \\ Jilin Agricultural University,Changchun 130118,China \\ 10821395@qq.com
}

\begin{abstract}
Keywords: Android mobile phone platform, experiment management, interaction
\end{abstract}
\begin{abstract}
This paper carries out the research on the experiment management applications which are based on the Android mobile phone platform. First of all, in this paper, the composition of experiment management system based on the Android mobile phone platform has been analyzed, and then the system function has been designed. At the same time, in this paper, the experiment management functions of the system have been implemented specifically, such as experiment booking, notification boards, laboratory real scenes and experimental instruction books, etc. Through the process of function implementation, the user interface is written by the Android technology on the Eclipse platform. The interaction is with Tomcat lightweight servers by the ways of GET and POST. The Servlet module is been used by the server to complete the login authentication and command response. Lastly, in this paper, the system test has been carried out, and the test results could be figured out by the Figure 4, Figure 5 and Figure 6 and Figure 7. The test results show that the implementation of experiment management system based on mobile phone platform could help the students and teachers to get the experiment booking and to obtain the curriculum changing notification easily and quickly. In addition, the module of laboratory real scenes could help the teachers to check the laboratory scenes in real time anytime and anywhere in the mobile terminal. The module of experimental instruction books is convenient for the students to preview experiments or to check during the experimental process, thus, the portability and efficiency of the experiment management work could be highly improved. At the same time, the experiment management system is of practicality and integrity which could meet the practical demands of experiment booking and experiment teaching notice. The interaction between teachers and students could be done anywhere and anytime. The workload of teachers has been lightened while the experiment practice for students is becoming convenient
\end{abstract}

\section{Introduction}

The experimental teaching is getting more and more attention with the rising requirements of higher education to the students' practical ability. The laboratory construction in colleges and universities plays a crucial role in cultivating students' practical ability and in improving students' ability of independent analysis, however, at present, the laboratory management system in colleges and universities are mostly the PC management system based on B / S mode, or even in the ways like teachers and students meeting together or paper registration, etc. which could not meet the demands in portability [1]. One laboratory may be used for different experiments of several courses and many grades of students, therefore, it is very important to allocate the experiment time and equipments reasonably in order to promote better communication between teachers and the students [2]. In this paper, research on experiment management application based on Android mobile phone platform has been carried out on the purpose of better utilization of laboratory resources and management efficiency improvement. Furthermore, the experiment management system has been designed and implemented with functions of experiment booking, notification boards, laboratory real scenes and experimental instruction books. 


\section{Research content}

\section{Composition experiment management system based on Android mobile platform}

The system is composed of 3 parts: the Android client, the backstage server and the database. The client is written by relative Android technology. The upper code which is the basis to complete the system functions is of Java code, it could provide the operation interface to users, be responsible for interaction with users and send related operational commands of users' to the sever [3]. The sever uses the Servlet technology to respond, the Web server uses Tomcat 6 as the Servlet container. The data storage is divided into local SQLite database and backstage MySQL database: the client uses SQLite database storage which uses data with high frequency but not frequently updated data. The server database is MySQL database, the sever accesses MySQL database through JDBC technology.

Functional designs of experimental management system based on Android mobile platform Android client functions

Through the client' $\mathrm{s}$ landing module, the user registration and system login could be accomplished and the system safety could be guaranteed [4]. Teachers and students could login after registration with their staff numbers and student IDs respectively, the system would verify their account passwords, and then corresponding navigation interface would pop out after successful access. In this system, the function modules have been humanized designed for the users of teachers and students. Besides the 2 interaction function modules of experiment booking and notification board, the laboratory real scenes function is provided for teachers and the experimental instruction book function is provided for students. The functional design is as shown in figure 1:

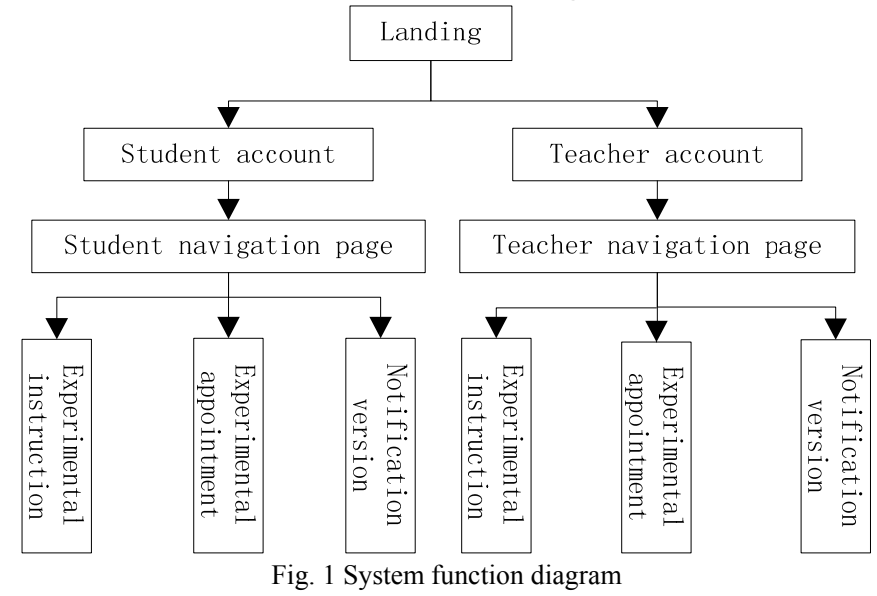

(1) Function design for teacher users

Teachers use the notification function to release the arrangement or temporary change of the curriculum $[5,6]$. Students use the notification board to check. The module of laboratory real scenes could help the teachers to assist students to complete the experiment with the laboratory assistant administrator, or, teachers could check the laboratory scenes in real time anytime and anywhere in the mobile terminal and save screenshots of the monitoring picture when a laboratory needs cleaning, equipment upgrade and maintenance.

(2) Function design for student users

The experimental instruction book is of outline design. After clicking the title of experiment, the experiment content, requirements, experiment equipment and process would be shown. It is convenient for the students to prepare and to check the experiments. The function of experiment booking is the crucial functional module in this system. The options after clicking in are: experiment content, date, class number and student number [7]. While choosing all the options, the booking information would be submitted to the server and would be displayed at the bottom of the careen. Teachers could save the booking audit information into the MySQL database through the booking check function. And the information would be sent to the client by the server and be displayed under the reservation information. The booking function process is as shown in figure 2 : 


\section{Server and database design}

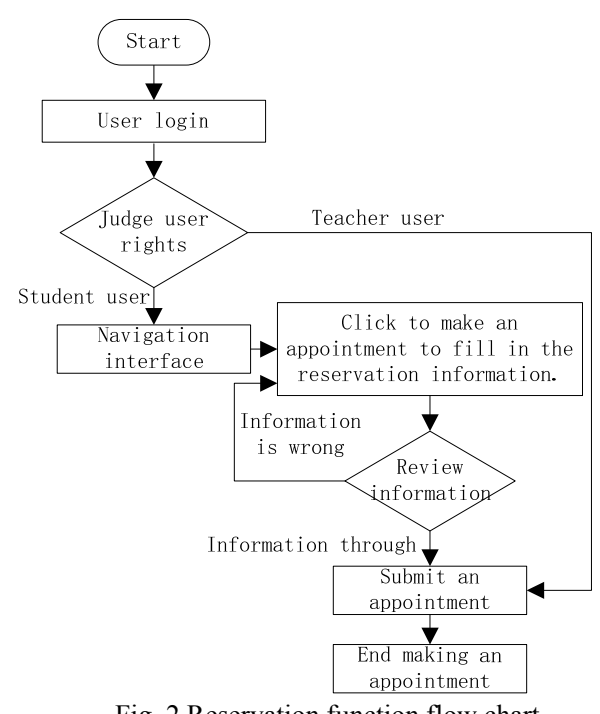

(1) Communication mode and Web server

Android provides the two interfaces--HttpURLconnection and HttpClient to access website procedure. This system uses the HttpClient interface development through 2 ways of GET and POST to interact with the server. The operation of GET is used to read information from the server. The operation of POST is used to modify and query the server contents. The client sends HTTP communication request to the specified URL server through the wireless network. The system server is in using of the Tomcat version 6.0 and is responded by the Servlet technology.

This system will select the way of GET or SET AA according to the request information of the client. The communication mode of each part of the system is as shown in the figure below:

(2) Database design

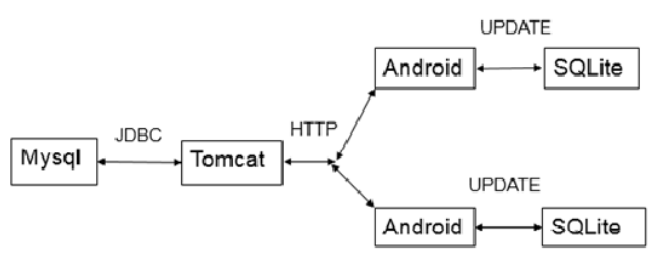

Fig.3 Communication mode of each part of the system

SQLite, the local small relational database, is responsible for the storage of the account information table, booking information and notice board information before updated. And it is of high efficiency to complete the functions of creating, deleting, adding, modifying and query by the 2 categories of SQLite Database and SQLiteOpenHelper. The database of MySQL is responsible for the data storage tasks of the user login validation, booking module and the notice board updated by teachers.

Web server interacts with the MySQL database through JDBC. It uses the Class. For Name method to load the drivers, then creates the Connection and Statement object, using the methods of execute Up-date or execute Query of the objects to execute the SQL statement, lastly, to obtain the message returned from the database by the Result Set object.

\section{Function implementation}

\section{Implementation of Android client}

Android client user interface is a bridge between the user and the software function. Before the programming, in the operating system, plug-in modules of Java Development Kit (JDK), Java integrated Development environment Eclipse, Soft-ware Development Kit and Android Development Tools (ADT) should be installed. The writing and debugging of the client is by the AVD management to create Device Android Virtual (AVD) on the computer.

The client interface includes: login interface, navigation interface and interfaces of each function. This system uses several basic controls like linear layout Lin-ear Layout, relative layout Relative Layout, and Text View, Edit Text, Button, Image View of the Android interface design, while, the 
advanced controls of Spinner and List-View. Code is written by Java language. The mechanism of Android Intent is used into interfaces switch.

\section{The implementation of main function modules}

(1) The login module

Firstly, the use of Relative Layout relative layout, adding 2 user names, inputting password into control Text View, setting the size and location, ands adding 2 Button buttons of login and register should be done. After that, the monitor events of the button in the Java files should be set through the on click () method, and the input user name and password should be matched to the backstage MySQL database. If the matching is a success, it would switch to related navigation interface through Intent (intention) mechanism, otherwise, the error messages would be printed by the make Text( ) method of the Toast category.

(2) Modules of reservation function and notification board

These two functions are of the most importance of this system. Through which experiment reservation could be made fast and conveniently between teachers and students. It no longer needs to meet face to face or through the Web experiment management system to operate, which fits the trend of mobile Internet era. When operating related functions, the HTTP request from the client is sent to the sever, and after data processing of the server, it would return to the XML files data flow. The client would analysis the data flow for display after receiving it.

(3) Module of laboratory outline

The folding catalogue design is used combining with the Android controls of linear layout, Text View and List View, etc. It would unfold when clicking every experiment project and fold when clicking again with related display disappears. Its data is stored in the local SQLite database.

(4) Module of laboratory real scenes

The commuter which connects to surveillance cameras must have networking capabilities. The implementation is as follows:

1) Firstly, a Java interfaces: Camera-Source should be created to define the connection Camera interface. The image data code is obtained by connecting remote Server through Http method. Then, the Java class: Camera implementation interface Camera Source should be created and the callback function onPreviewCallback () should be created within.

2) The Socket monitoring program is writing in the server. The JSON data is sent to the client at any time after the start of the thread.

3) Data received by the client is done the NV21 decoding. The design of Android interface is required to create Activity to configure the IP connecting to the camera computer, ports and monitor screenshot saving location. After the corresponding XML file configuration and logic code compilation, the laboratory camera could be connected to check the laboratory real scenes. If the connection successes once, by defining a tool category Database Helper to encapsulate the SQLite operation, the server information will be saved in the SQLite database for future use.

(5) Implementation of the experimental instruction book function for student users

Combining with the Android controls of linear layout, Text View and List View, etc., the experimental instruction book is designed in folding catalogue design. It would unfold when clicking every experiment project and fold when clicking again with related display disappears. The experimental instruction book includes: basic experiment of interchanger, basic experiment of router, VLAN configuration experiment, RIP routing choice experiment, diagnosis experiment of network test box, FTP and Web server configuration experiment, firewall application experiment. After clicking on the title of an experiment, the secondary title would be displayed: the purpose and steps of the experiment, the principle and inspiration of the experiment.

\section{Test results and discussion}

In order to confirm the validity of the system, we have done tests respectively on each function of the system. The first test is to the functions of login and registration. App is running on the Android 
emulator (AVD), the landing interface is opened with double-click, as shown in figure 4. If the backend server authentication is successful, then users could be into the navigation interface.

Operation tests are done on each function of the experiment booking, notification board and laboratory real scenes to check the response speed of system, interactive data accuracy and the functional integrity. Experiment booking function display is as shown in figure 5. The experiment project can be set according to specific circumstances after clicking the dropdown menu of all the options of the experiment lesson, month, day, date, number, then selecting, submitting and waiting for the approval of teachers. The submitted information will be displayed below. The notice information issues by teacher users would be shown on the notification board. Clicking the update button could get the latest information. It is as shown in figure 6 .

After entering into the laboratory real scenes function for teacher users, the live-action connecting configuration is done. The precondition of this connection is that the computer connecting to the laboratory camera is accessing to the Internet. The real scenes of the laboratory will be displayed after success linking. It is as shown in Figure 7.

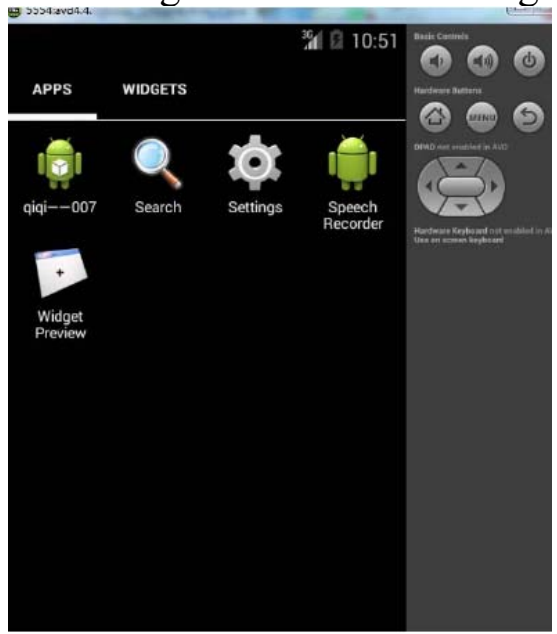

Fig.4 The client display in the simulator

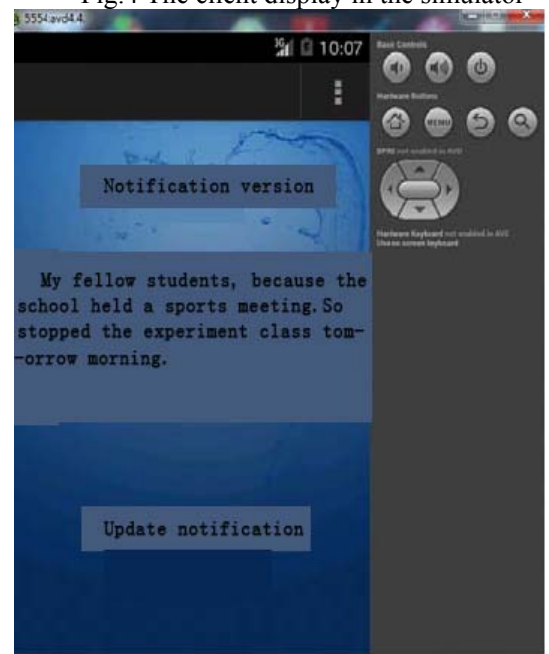

Fig.6 Function set of laboratory real scenes

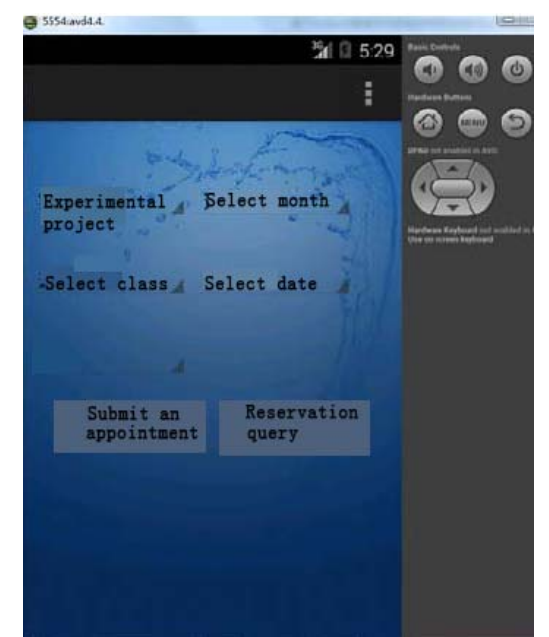

Fig.5 Interface of experiment booking

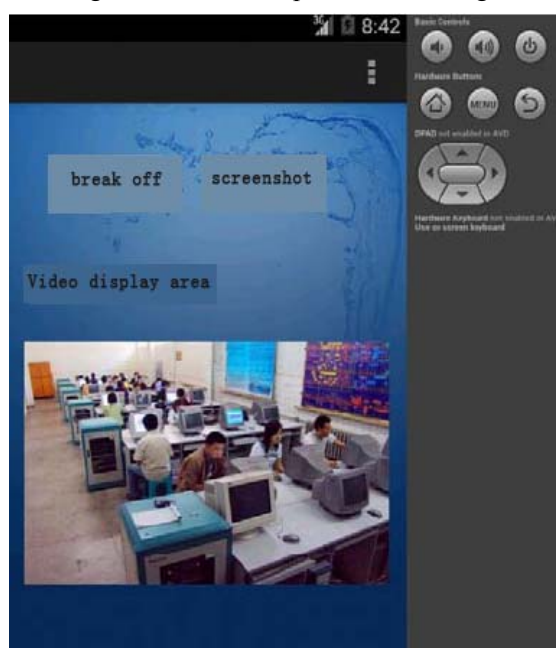

Fig.7 Real scenes of laboratory

Test results show that: in the registration function and the notification board function, interaction between teacher user and student users is good. Quick response of the backend server, stability of the database could well support to complete the experiment registration function and the notification function. The function of laboratory real scenes for teacher users could connect to the camera in the laboratory and display the real scenes of the laboratory. The configuration interface parameter information could be successfully saved. There is no need to input the configuration parameters when users login again. In the process of displaying, it is smooth enough to take screenshots and to save the pictures into the defined folder of the SD card. Experimental information in the student users' experimental instruction book could be opened, folded and displayed without any bug. 


\section{Conclusion}

Based on the above results, we can find that the experiment management system which is basing on mobile phone platforms could help both teachers and students to complete the booking and to obtain course change notification fast and conveniently. The module of laboratory real scenes could help the teachers to check the laboratory scenes in real time anytime and anywhere in the mobile terminal. The module of experimental instruction books is convenient for the students to preview experiments or to check during the experimental process, thus, the portability and efficiency of the experiment management work could be highly improved. At the same time, the experiment management system is of practicality and integrity which could meet the practical demands of experiment booking and experiment teaching notice. The interaction between teachers and students could be done anywhere and anytime. The workload of teachers has been lightened while the experiment practice for students is becoming convenient.

\section{References}

[1] Wang H, Zheng P, Xia L, et al. Design of mobile experimental system based on Android[J]. Experimental Technology \& Management, 2015.pp: 12-19

[2] Wang Y. The Application of Experimental Research Method in the Overseas Management Accounting Research:Based on 73 Literatures[J]. Journal of Nanjing Audit University, 2013.pp:31-42.

[3] Jahns J, Edelmann A, Helfert S, et al. Distance teaching and learning in photonics: a 10-year experiment $[\mathrm{C}] / /$ Education and Training in Optics and Photonics: ETOP 2015. International Society for Optics and Photonics, 2015.pp:12-17

[4] Meier R. Professional Android 4 Application Development[M]. Wrox, 2012.

[5] Hongbo Y U, Dai L, Aiping L I, et al. Android Application Development in 24 Hours[M]. Sams, 2013.pp:7-9.

[6] Reddy S V, Tamalampudi S, Deshpande P, et al. Systems and methods for application development: US, US8910115[P]. 2014.

[7] Ali Z, Ismail R. Design and development of Android mobile application for students of engineering education in Saudi Arabia[C]// Information Society (i-Society), 2013 International Conference on. IEEE, 2013:228-233. 